\title{
A Survey to Identify Determinants That Influence Self-Perceived Sensitive Skin in a British Population: Clues to Developing a Reliable Screening Tool for Sensitive Skin
}

\author{
Neil Fawkes (1D \\ Evi Tselenti \\ Neil Shah \\ Victoria Lappin \\ Neil Smith \\ Arjun Narasimhan \\ Adam B Smith
}

Reckitt Benckiser Health Care Ltd., Hull, HU8 7DS, UK
Correspondence: Neil Fawkes Reckitt Benckiser Health Care Ltd., Dansom Lane, Hull, HU8 7DS, UK Email neil.fawkes@rb.com
Background: Skin sensitivity characteristics and triggers have been identified in populations in previous studies. However, few have compared these characteristics among selfreported sensitive skin.

Objective: The aim of the study was to evaluate and compare specific intrinsic and extrinsic triggers of skin sensitivity between individuals with self-reported sensitive skin and nonsensitive skin.

Methods: A systematic literature review was undertaken to identify intrinsic and extrinsic factors associated with sensitive skin. A 167-item survey was developed on the basis of the literature review. The survey was completed online by a sample of adult participants drawn from the general United Kingdom population. Participants also completed sociodemographic and self-reported health questions.

Results: A total of 3050 surveys were completed: 1526 participants with self-reported skin sensitivity and 1524 participants not reporting skin sensitivity. There was a decrease in selfreported skin sensitivity with increasing age $(\mathrm{p}<0.05)$, and proportionally more women reported sensitive skin. Smoking also led to a higher frequency of sensitive skin. All signs and symptoms of sensitive skin, such as itch, dryness/flakiness, roughness and flushing/ blushing were more commonly reported by those with self-reported sensitive skin. These were frequently reported in association with external factors (cold/windy weather, clothes and fabrics), as well as internal factors such as pre-existing skin conditions and atopy.

Conclusion: The study evaluated self-reported sensitive skin against a non-sensitive skin in order to identify common inherent and external triggers to distinguish between these groups in a large general population study in the United Kingdom. The key symptoms and signs of this syndrome identified in the literature were confirmed to be reported significantly more when compared with those without sensitive skin. However, no correlation or pattern of symptomology could be identified, reinforcing the complexity of this condition. Given the strong differentiation from the non-sensitive group, the results of this research could be utilised for the development of a clinically meaningful screening tool.

Keywords: sensitive skin, smoking, age, asthma, atopy

\section{Introduction}

Sensitive skin is a common condition that affects an estimated $50 \%$ of the individuals ${ }^{1}$ with global reported prevalence rates ranging from $12.79 \%$ to $68.4 \% .^{2-4}$ Several 
international surveys have shown an increase in self-reported sensitive skin prevalence over the last 5 years, particularly in men. ${ }^{5-8}$ This suggests potential changes in population skin characteristics and attitudes to self-reported skin sensitivity.

Despite the high prevalence of skin sensitivity, there is a lack of clear consensus regarding its definition and diagnosis of people with sensitive skin. A recent expert consensus of sensitive skin suggests that it is a complex problem characterised by unpleasant sensations (stinging, burning, pain, pruritus, and tingling sensations) in response to stimuli that normally should not provoke such sensations. These unpleasant sensations cannot be explained by lesions attributable to any skin disease. The skin colour can appear normal or be accompanied by erythema. Sensitive skin can affect all body locations, especially the face. ${ }^{9}$ The development of cosmetic products routinely includes pre-market product testing intended to ensure that any marketed product is free of irritant potential and often if they are suitable for people with sensitive skin. Nonetheless, it is not uncommon for companies to receive post-marketing complaints of unpleasant reactions to such products. ${ }^{10}$ This is also reflected in existing epidemiology studies, for instance, where $78 \%$ of the consumers that declared "very sensitive skin" avoided specific products due to prior experiences of unpleasant effects with their use. ${ }^{11}$

The reliable diagnosis of sensitive skin remains problematic. A variety of methods have been used to identify individuals with sensitive skin, most of which have significant limitations. ${ }^{12}$ For instance, tests have focused on eliciting specific objective sensory reactions, such as stinging or burning, or provoking irritation with enhanced means of detecting inflammatory responses, which are often unpleasant for participants. That said, there are tools available to determine the severity of sensitive skin, such as the Sensitive Scale $-10 .{ }^{13}$ Although enhanced detection methods may be able to identify irritation reactions, there is (to the authors' knowledge) no reliable screening instrument to determine the presence of sensitive skin for potential participants in clinical cosmetic studies.

The aims of this research were therefore to evaluate the characteristics and identify determinants of self-reported sensitive skin in order to provide the framework for the development of a screening tool, which can consistently identify patients and/or consumers with the common features of self-identified skin sensitivity.

\section{Methods}

\section{Survey Development}

A structured literature review was conducted to establish a firm understanding of sensitive skin and to conceptualise the required questionnaire content. The search strategy identified existing sensitive skin questionnaire content and established the intrinsic/extrinsic triggers and symptoms experienced by subjects with sensitive skin types. The following search terms were used:

('sensitive skin'/exp OR 'sensitive skin') AND ([adult]/ $\lim$ OR [middle aged]/lim OR [aged]/lim OR [very elderly]/lim).

The following electronic databases were utilised: Embase (1974 to present); MEDLINE (1946 to present, incorporating Epub Ahead of Print, In-Process \& Other Non-Indexed Citations, MEDLINE Daily); EBM Reviews (incorporating: HTA database, The National Health Service Economic Evaluation Database (NHS EED), Cochrane Central register of Controlled Trials (CENTRAL), Database of Abstracts of Reviews of Effects (DARE)).

The following sources were hand searched to supplement the findings from the electronic databases:

-Reference lists of included studies.

- Google Scholar to identify relevant documents not identified by the database search, including:

Regulatory guidance; National and international guidance/position papers; Publicly available reports from professional bodies; Public access dossiers; Book chapters; Clinical trial registries; US NIH registry and results database (https://clinicaltrials.gov); WHO ICTRP registry (http://apps.who.int/trialsearch/).

The eligibility criteria are shown in Supplementary Table 1. The PRISMA (Preferred Reporting Items for Systematic Reviews and Meta-Analyses) flow diagram ${ }^{14}$ is shown in Figure 1.

\section{Literature Review}

The literature search identified 4548 potential articles. Following abstract screening, 52 articles were deemed eligible for the full-text evaluation and were subsequently included in the final literature review (Figure 1). Supplementary Table 2 summarises the major demographic, symptomology, and environmental triggers associated with sensitive skin.

A series of clinically relevant items were identified as factors indicated in sensitive skin types by the reviewers. These items were developed into a 167-item questionnaire, 


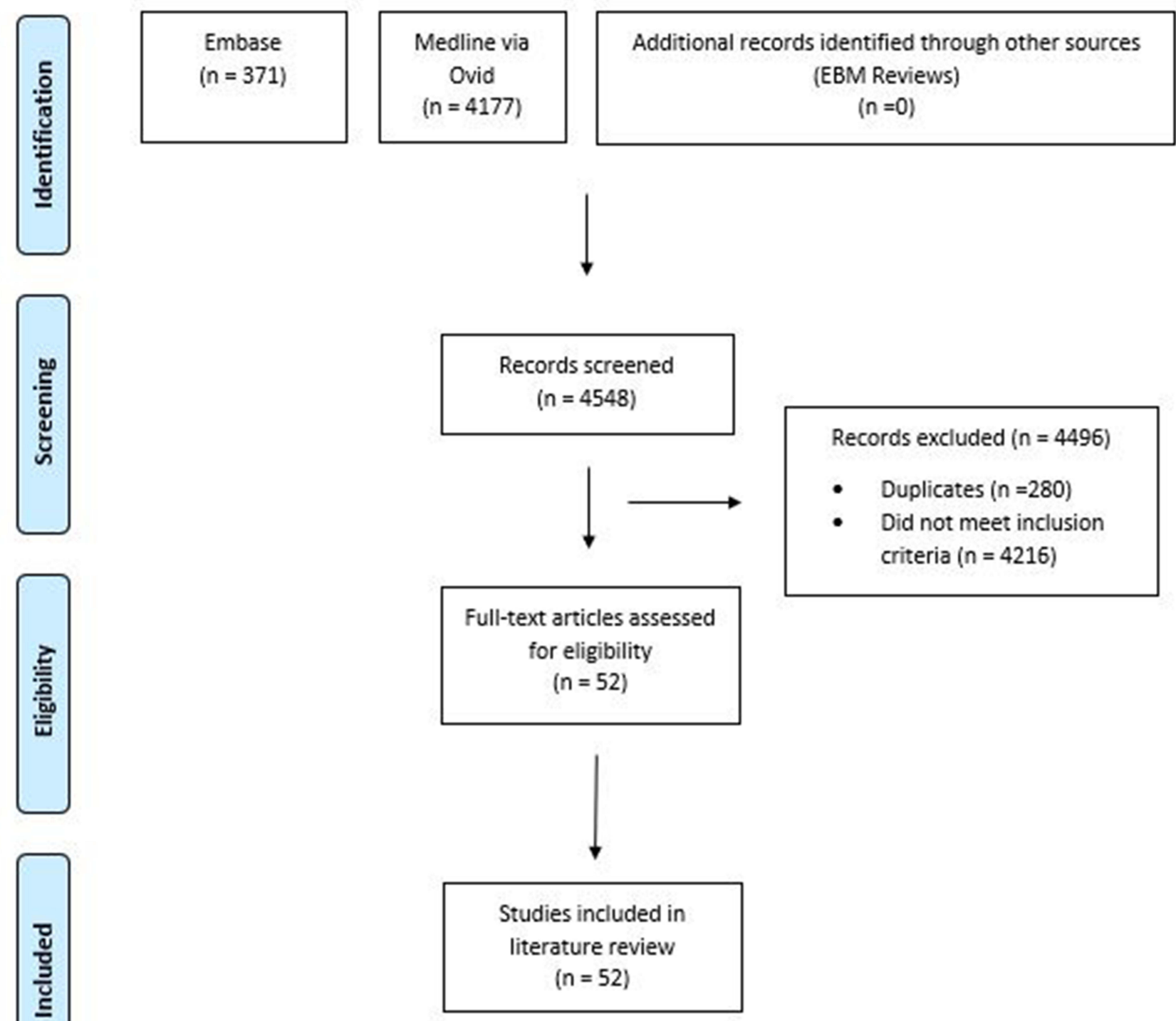

Figure I Literature review: PRISMA flow diagram.

which included a regional assessment (face/body and scalp) of neurosensory and physical responses to extrinsic and intrinsic triggers. These questions, alongside a selection of sociodemographic and health-related questions, were presented to participants in the survey.

\section{Survey Sample}

Potential respondents were invited to participate from an online panel maintained by a third-party data collection and analytics provider (Qualtrics LLC). The study sample included males and females over the age of 18 years who resided in the United Kingdom (UK). The target population was participants who self-reported sensitive skin, as well as those with non-sensitive "normal" skin. No formal sample size calculation was undertaken; however, in order to ensure the sample was as representative of the UK adult population as possible (in terms of geographical distribution between the four home nations and gender). The study aimed to recruit 3000 participants with a minimum of 1500 participants with self-reported sensitive skin and 1500 with selfreported non-sensitive skin. Survey participants receive a modest number of tokens in compensation for their time, which could be collated and converted into online vouchers (eg, Amazon). Non-English-speaking participants or 
participants unable to read English were excluded from study participation.

\section{Ethical Considerations}

As potential participants were not patients (or healthcare practitioners) recruited through the UK National Health Service (NHS) this study was exempt from approval by an NHS Research Ethics Committee. Nevertheless, ethical considerations outlined in the good practice in internetmediated research ${ }^{15}$ were applied to this study. Prior to participating in the study, potential participants were presented with an introduction to the study explaining the nature and sponsor of the study (Reckitt Benckiser, RB), as well as the study sponsor's data management policy. No content deemed to be either sensitive or potentially distressing to participants was presented. All participants were provided informed consent prior to completing the survey, including their right to withdraw from the study at any stage. It should also be noted that participants had already consented to be approached about study participation (not just in the current study) through their registration as panel participants. The study was conducted in compliance with the Declaration of Helsinki.

\section{Data Processing and Statistical Analysis}

The survey data were exported into MS Excel from the Qualtrics platform. Descriptive statistics included frequency, mean and standard deviation. Continuous variables (eg, mean differences between groups) were analysed with independent sample $t$-tests. Categorical variables were assessed using chi-squared tests (for trend). Associations between symptoms and signs of sensitive skin were evaluated using Spearman's rho. Statistical significance was set at $\mathrm{p}<0.05$. The open-source statistical software R (version 3.5.2) was used for the analysis. ${ }^{16}$

\section{Results}

\section{Survey}

A total of 3050 participants completed the surveys: 1526 and 1524 participants who reported, respectively, sensitive skin and non-sensitive skin. Two responders, however, were omitted from the analysis; one participant reported smoking $>2000$ cigarettes per day (self-reported sensitive skin), and the other participant reported their age as 109 years (no self-reported sensitive skin). A summary of the demographics is provided in Table 3 . The mean time taken to complete the survey was 11 minutes (range: 2-601 minutes, standard deviation, SD: 18.83 minutes).

\section{Skin Sensitivity, Gender and Age}

Women were more likely to report sensitive skin than men, whereas men more commonly reported non-sensitive skin (Table 1). These differences were statistically significant $(p=0.007)$. Participants who preferred not to provide gender were not included in this particular analysis due to small numbers $(\mathrm{N}=13)$.

The average age of the sample was 42.01 years (range: 18 86 years). Participants with self-reported sensitive skin were on average younger than those not reporting sensitive skin (39.28 years (range: $18-86$ years) and 44.73 years (range: $18-$ 81 years $)$, respectively $(t(1,3046)=10.28, \mathrm{p}<0.0001)$.

Participants were stratified into groups according to age $^{17}$ (Table 1). The younger age groups reported higher rates of sensitive skin in comparison to non-sensitive skin. The proportion of participants reporting sensitive skin increased up to the age of 34 years (61.9\%), after which self-reported sensitive skin reduced in prevalence. The age of participants increased with the lowest levels of sensitive skin $(28.3 \%)$ reported by the oldest age group (65 to 79 years $)$. This trend was statistically significant $(\mathrm{p}<0.0001)$. Participants aged $>80$ years were removed from this analysis due to low numbers $(\mathrm{N}=5)$. For each age category up to category $65-79$, there were proportionally more women reporting sensitive skin compared to men $(\leq 24: 75.4 \%$ vs $24.6 \%$; 25-34: $72.1 \%$ vs $27.9 \%$; 35-49: $63.4 \%$ vs $36.6 \%$; 50-64: $65.3 \%$ vs $34.7 \%$; and, $65-79$ : $48.0 \%$ vs $52.0 \%$ ). However, none of these differences were statistically significant.

\section{Skin Sensitivity and Ethnicity}

The ethnicities of the self-reported sensitive and nonsensitive skin groups are presented in Table 1. A large proportion of the sample reported a White English/Welsh/ Scottish/Northern Irish/British ethnicity and other white background. Due to the small numbers recruited in the other the groups, the data did not permit analysis.

\section{Skin Sensitivity, Fitzpatrick Phototype, Smoking Status, Skin Conditions and Atopy Fitzpatrick Phototype}

Participants with Fitzpatrick phototypes I and III reported sensitive skin more frequently than non-sensitive skin, and 
Table I Demographics of Survey Population

\begin{tabular}{|c|c|c|c|c|c|}
\hline & $\mathbf{n}$ & $\begin{array}{c}\text { Subjects with Sensitive } \\
\text { Skin n (\%) }\end{array}$ & $\begin{array}{c}\text { Subjects with Non-Sensitive } \\
\text { Skin n (\%) }\end{array}$ & $\begin{array}{c}\chi^{2} \text { Value (Degrees of } \\
\text { Freedom) }\end{array}$ & $P$ value \\
\hline Total participants (\%) & 3048 & $1525(49.9)$ & $1523(50.0)$ & & \\
\hline \multicolumn{6}{|l|}{ Gender $(\mathrm{N}, \%) \mathrm{I}$} \\
\hline Male & 1071 & $500(32.8)$ & $57 \mid(37.5)$ & & \\
\hline Female & 1964 & $1017(66.7)$ & $947(62.2)$ & $7.56(1)$ & 0.006 \\
\hline Prefer not to say & 13 & 8 & 5 & & \\
\hline \multicolumn{6}{|l|}{ Age Group (years) (N, \%) } \\
\hline$\leq 24$ & 390 & $213(14.0)$ & $177(\mid 1.6)$ & & \\
\hline $25-34$ & 706 & $437(28.7)$ & $269(17.7)$ & & \\
\hline $35-49$ & 990 & $519(34.0)$ & 47। (30.9) & & \\
\hline $50-64$ & 692 & $278(18.2)$ & $414(27.2)$ & & \\
\hline 65-79 & 265 & $75(4.9)$ & $190(12.5)$ & & \\
\hline$\geq 80$ & 5 & 3 & 2 & $89.87(\mathrm{I})$ & $0.0001 *$ \\
\hline \multicolumn{6}{|l|}{ Ethnicity ( $\mathrm{N}, \%)$} \\
\hline African & 25 & 8 & $17(1.1)$ & & \\
\hline Any other Asian background & 11 & 6 & 5 & & \\
\hline Any other black/African /Caribbean & 5 & 3 & 2 & & \\
\hline background & & & & & \\
\hline Any other ethnic group & 18 & 9 & 9 & & \\
\hline Any other white background & 115 & $67(4.4)$ & $48(3.2)$ & & \\
\hline Arab & 5 & 3 & 2 & & \\
\hline Bangladeshi & 18 & $10(0.7)$ & 8 & & \\
\hline Caribbean & 18 & $12(0.8)$ & 6 & & \\
\hline Chinese & 30 & $14(0.9)$ & $16(1.1)$ & & \\
\hline Gypsy or Irish traveller & 5 & 4 & 1 & & \\
\hline Indian & 47 & $21(1.4)$ & $26(1.7)$ & & \\
\hline Irish & 33 & $25(1.6)$ & 8 & & \\
\hline Pakistani & 44 & $24(1.6)$ & $20(1.3)$ & & \\
\hline White and Asian & 29 & $14(0.9)$ & $15(1.0)$ & & \\
\hline White and black African & 15 & 6 & 9 & & \\
\hline White and black Caribbean & 20 & $13(0.9)$ & 7 & & \\
\hline Total & 2589 & $1273(83.5)$ & $1316(43 \%)$ & & \\
\hline \multicolumn{6}{|l|}{ Fitzpatrick Skin Type (N, \%) } \\
\hline $\mathrm{I}-\mathrm{I}$ always burn, do not tan & 424 & $273(17.9)$ & $|5|(9.9)$ & $35.104(1)$ & 0.0001 \\
\hline II-I always tan after initial burn & 697 & $332(21.8)$ & $365(24.0)$ & $1.56(1)$ & 0.211 \\
\hline III-I burn easily, tan poorly & 851 & $483(31.7)$ & $368(24.2)$ & $15.54(1)$ & 0.0001 \\
\hline IV-I burn minimally, tan easily & 630 & $269(17.6)$ & $361(23.7)$ & $13.435(1)$ & 0.0002 \\
\hline V-I rarely burn, tan darkly & 149 & $51(3.3)$ & $98(6.4)$ & $14.826(I)$ & 0.0001 \\
\hline VI-I never burn, always tan & 297 & $117(7.7)$ & $180(11.8)$ & $13.364(1)$ & 0.0002 \\
\hline \multicolumn{6}{|l|}{ Smoking Status (N, \%) } \\
\hline Non smoker & 1564 & $750(49.2)$ & $814(53.5)$ & $2.61(1)$ & 0.10 \\
\hline Current smoker & 781 & $440(28.9)$ & $34 \mid(22.4)$ & $12.54(I)$ & 0.0003 \\
\hline Previous smoker & 703 & $335(22.0)$ & $368(24.2)$ & $1.54(1)$ & 0.21 \\
\hline \multicolumn{6}{|l|}{ Dermatology Condition ( $\mathrm{N}, \%)$} \\
\hline Dermatitis (allergy or irritant) & 402 & $330(21.6)$ & $72(4.7)$ & & \\
\hline Eczema & 561 & $456(29.9)$ & $105(6.9)$ & & \\
\hline Rosacea & 171 & $134(8.8)$ & $37(2.4)$ & & \\
\hline Acne & 472 & $343(22.5)$ & $129(8.5)$ & & \\
\hline Psoriasis & 244 & $185(12.1)$ & $59(3.9)$ & & \\
\hline Contact urticaria & 57 & $49(3.2)$ & 8 & & \\
\hline Dry skin & 1158 & $823(54.0)$ & $335(22.0)$ & & \\
\hline
\end{tabular}

(Continued) 
Table I (Continued).

\begin{tabular}{|c|c|c|c|c|c|}
\hline & $\mathbf{n}$ & $\begin{array}{c}\text { Subjects with Sensitive } \\
\text { Skin n (\%) }\end{array}$ & $\begin{array}{c}\text { Subjects with Non-Sensitive } \\
\text { Skin n (\%) }\end{array}$ & $\begin{array}{c}\chi^{2} \text { Value (Degrees of } \\
\text { Freedom) }\end{array}$ & $P$ value \\
\hline Ichthyosis vulgaris & 0 & 0 & 0 & & \\
\hline Keratosis pilaris & 64 & $45(3.0)$ & $19(1.3)$ & & \\
\hline None of these & 1227 & $254(16.7)$ & $973(63.9)$ & & \\
\hline \multicolumn{6}{|c|}{ History of Atopy (N, \%) } \\
\hline Atopic & 1429 & 911 (59.7) & $518(34.0)$ & & \\
\hline Asthma & 616 & $395(25.9)$ & $221(14.5)$ & & \\
\hline Hay Fever & 1181 & $780(51.2)$ & $401(26.3)$ & & \\
\hline Not Atopic & 1619 & $614(40.3)$ & $1005(6.6)$ & & \\
\hline \multicolumn{6}{|c|}{ Family History Atopy (N, \%) } \\
\hline Atopic & 1831 & $623(40.9)$ & I208 (79.3) & & \\
\hline Eczema & 842 & $571(37.4)$ & $271(17.8)$ & & \\
\hline Hay Fever & 1181 & $780(5 \mid .2)$ & $40 \mathrm{I}(26.3)$ & & \\
\hline Asthma & 616 & $395(25.9)$ & $221(14.5)$ & & \\
\hline Not Atopic & 1217 & $403(26.4)$ & $814(53.5)$ & & \\
\hline
\end{tabular}

Notes: Bold $\mathrm{P}$ values represent statistical significance. ${ }^{*}$ Chi-squared test for trend. ${ }^{\mathrm{i}}$ Column percentages are presented in the table where $\mathrm{N}>\mathrm{I} 0$.

phototypes IV, V, and VI more frequently identified as having non-sensitive skin vs sensitive skin (Table 1).

\section{Skin Sensitivity and Smoking}

Current smokers reported sensitive skin more frequently than non-sensitive skin; conversely, non-smokers reported non-sensitive skin more frequently than sensitive skin $(56.3 \%$ vs $43.7 \% \mathrm{p}=0.0003$ and $52.0 \%$ vs $43.7 \% \mathrm{p}=$ 0.10 , respectively; Table 1). In the previous smoker group, non-sensitive skin was more commonly reported than sensitive skin $(52.3 \%$ vs $47.7 \% \mathrm{p}=0.21)$.

Within the current smoker group, those smoking tobacco products for less than 10 years reported sensitive skin more frequently than non-sensitive skin. Participants who had smoked for 11 years or more reported nonsensitive skin more frequently than sensitive skin. The differences between the groups were however not statistically significant, and no statistically significant differences were identified between calculated pack-years and reported sensitive/ non-sensitive skin type.

\section{Skin Sensitivity, Skin Conditions and Atopy}

Self-reported skin sensitivity is shown by current dermatological conditions and atopy in Table 1. In general, having preexisting dermatological conditions and history of atopy is more likely to also lead to self-reported skin sensitivity. For instance, a total of 1833 participants reported one or more dermatological condition, $69.5 \%(\mathrm{~N}=1274)$ of these participants also reported sensitive skin (odds ratio, OR 8.75, 95\% confidence interval (CI): 7.38 to 10.38).

A similar pattern was also observed in terms of atopy: participants with self-reported sensitive skin are more likely to have a history of atopy than those with nonsensitive skin: OR 2.88 (95\% CI: 2.48 to 3.34 ).

\section{Symptoms and Signs of Sensitive Skin: Body, Face and Scalp}

All symptoms and signs were reported in the sensitive skin group more frequently than the non-sensitive skin group on the face and body (Table 2). In the sensitive skin group, the most commonly reported symptom was itch. Dry/flakiness, skin roughness, and easy flushing/blushing were the most frequently reported signs $(\mathrm{p}<0.0001)$. The degree of association between symptoms, as well as between signs of sensitive skin tended to be low for those participants with self-reported sensitive skin (Table 3). For instance, the highest level of association was seen between "burning" and "stinging," which were experienced on the face (rho $=0.507, \mathrm{p}<0.001$ ) as well as the body (rho $=0.532, \mathrm{p}<0.001$ ). For signs, facial "redness" and "blush/flushing" showed the greatest degree of correlation ( $\mathrm{rho}=0.325, \mathrm{p}<0.001)$.

\section{Sensitive and Skin Type}

Participants with oily or combination skin types on the face reported sensitive skin more frequently than non-sensitive 
Table 2 Symptoms and Signs in Sensitive and Non-Sensitive Skin Groups on Both Face and Body

\begin{tabular}{|l|l|l|}
\hline Symptoms/Signs* & $\begin{array}{l}\text { Sensitive } \\
\text { Skin }\end{array}$ & $\begin{array}{l}\text { Non-Sensitive } \\
\text { Skin N (\%) }\end{array}$ \\
\hline Itch & $836(54.8)$ & $208(13.6)$ \\
Stinging & $414(27.1)$ & $71(4.7)$ \\
Burning & $34 I(22.4)$ & $62(4.1)$ \\
Tightness & $588(38.6)$ & $154(10.1)$ \\
Redness & $376(24.7)$ & $82(5.4)$ \\
Dry/flaky & $1068(70.0)$ & $467(30.6)$ \\
Roughness & $986(64.7)$ & $382(25.0)$ \\
Flushing/blushing & $958(62.8)$ & $556(36.5)$ \\
Hives & $396(26.0)$ & $101(6.6)$ \\
Swelling around the eyelids & $486(31.9)$ & $192(12.6)$ \\
\hline
\end{tabular}

Notes: *All contrasts between two groups statistically significant at $p<0.000$ I (Chisquared test).

skin. Participants with a normal/dry skin type (with possible scaling) on the face reported non-sensitive skin more frequently than an overall sensitive skin type (Table 4). Participants with oily skin on the body reported sensitive skin more frequently than non-sensitive skin. Normal/dry skin types (with possible scaling) on the body reported nonsensitive skin more frequently than sensitive skin. Participants with combined (dry and oily) skin on the body reported similar frequencies of sensitive and non-sensitive skin. Participants with a different skin type in the face and body region reported sensitive skin more frequently than non-sensitive skin.

\section{Sensitive Skin: Extrinsic and Intrinsic} Factors

Both symptoms and signs were reported consistently and more frequently by participants with self-reported sensitive skin than those with non-sensitive skin for both extrinsic factors (cold/windy weather; hot/dry weather; sun; clothes/fabrics) and intrinsic factors (stress and pre-existing skin conditions). The results for the intrinsic factors are shown in Supplementary Table 3. These differences were most apparent for cold/windy weather, where facial itch (54.8\%) and facial redness $(51.7 \%)$ were the most frequently reported individual symptoms of sensitive skin (Supplementary Table 3). Dry/flaky skin (58.9\%) and skin roughness (56.5\%) were also commonly reported signs on both face and body under these external conditions. Facial and body itch were also most frequently reported individually under other extrinsic conditions, such as hot/dry weather $(41.97 \%, 43.6 \%)$, and sun $(37.2 \%, 37.3 \%)$. All signs and symptoms were more prevalent in the sensitive skin group when evaluated by pre-existing skin conditions, particularly itch (69.2\%), dry/flaky skin (65.4\%) and skin roughness (59.2\%). Itch was also the most often reported symptom associated with stress (45.3\%) (Supplementary Table 4).

Table 3 Correlations Between Symptoms and Signs for Self-Reported Sensitive Skin

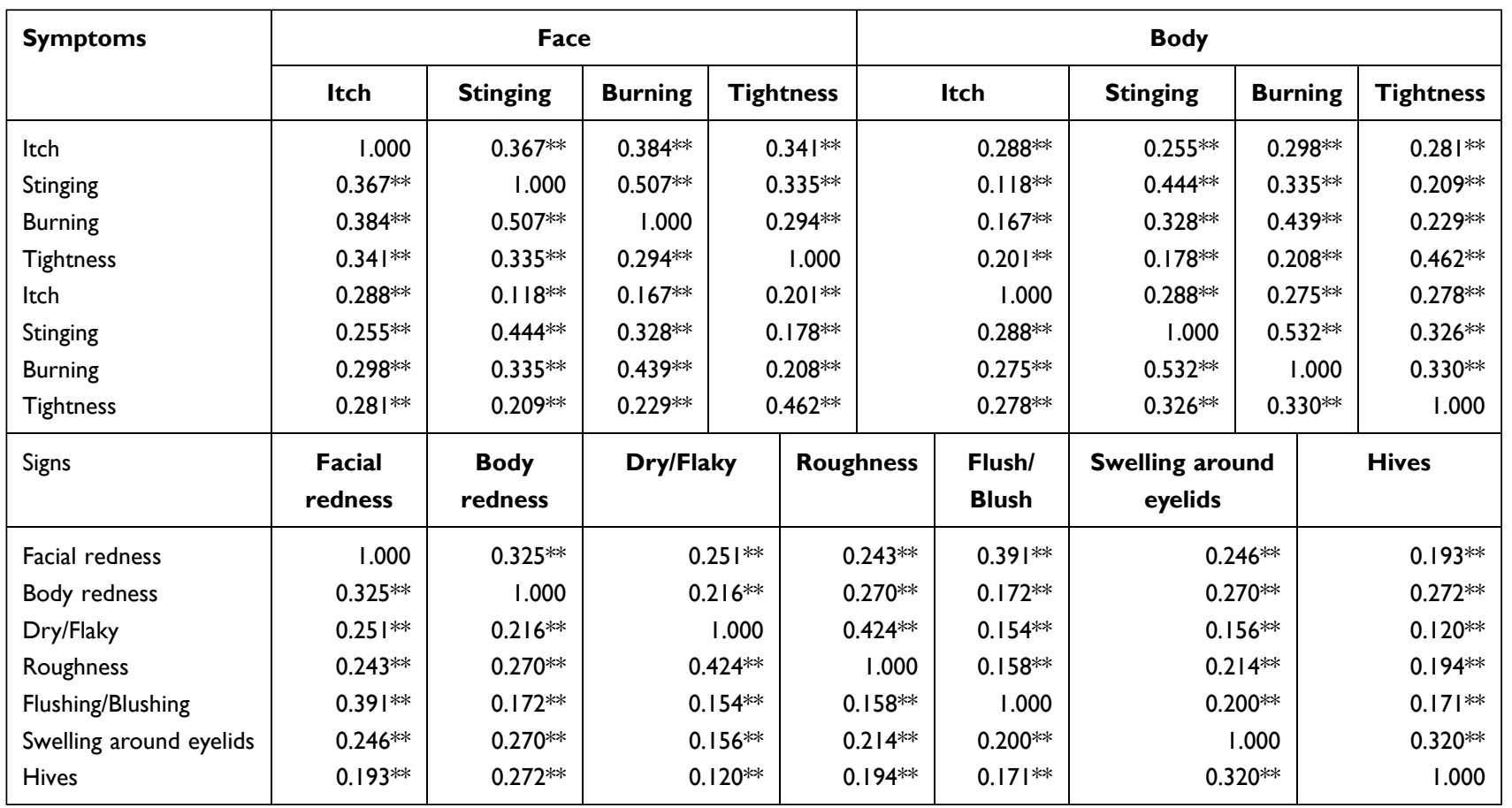

Note: $* * \mathrm{p}<0.01$ (Spearman rank correlation). 
Table 4 Sensitive Skin and Skin Type

\begin{tabular}{|l|c|c|c|c|c|c|}
\hline \multirow{2}{*}{ Symptom* } & \multicolumn{3}{|c|}{ Sensitive Skin (n= 1525) } & \multicolumn{3}{c|}{ Non-Sensitive Skin (n=1523) } \\
\cline { 2 - 7 } & Face (N, \%) & Body (N, \%) & Face and Body (N, \%) & Face (N, \%) & Body (N, \%) & Face and Body (N, \%) \\
\hline Oily & $256(8)$ & $194(5)$ & $102(3)$ & $229(8)$ & $143(6)$ & $82(3)$ \\
Combined (dry and oily zones) & $580(19)$ & $299(10)$ & $187(6)$ & $504(17)$ & $269(9)$ & $173(6)$ \\
Normal/Dry (can have scaling) & $689(23)$ & $1032(34)$ & $555(18)$ & $790(26)$ & $1111(36)$ & $709(23)$ \\
\hline
\end{tabular}

Note: ${ }^{*}$ All contrasts between two groups statistically significant at $p<0.0001$ (Chi-squared test).

\section{Discussion}

The results of this large representative UK study demonstrated significant differences in key symptoms (itch, stinging, burning, tightness) and signs (dry/flaky, roughness, blushing) of skin sensitivity between participants self-reporting sensitive skin and those reporting normal skin. In addition, the results also highlighted the demographic, extrinsic, and intrinsic factors underlying these differences.

Sensitive skin is a commonly used term by patients and clinicians, as well as the cosmetic industry. This syndrome represents a complex clinical challenge faced by dermatologists and other skin care professionals. Patients with sensitive skin often present subjective complaints that can vary widely depending on triggering factors and patient characteristics. ${ }^{18}$ Biophysical evaluations such as calorimetry, transepidermal water loss (TEWL), and capacitance have been used to establish objective differences between sensitive and non-sensitive populations with inconsistent results. Furthermore, the application of these methodologies is limited by the absence of validated questionnaires to identify and distinguish a population, which self identifies as having sensitive skin. ${ }^{10,19}$

This complexity reinforces the need for a screening algorithm to determine a diagnosis. Few studies have compared patient characteristics among self-reported sensitive skin and self-reported non-sensitive skin groups. This may give clues to advance our understanding of sensitive skin and to help in the development of a screening tool. The results of this study correspond with the epidemiological data, ${ }^{5-8}$ in which men appear to be closing the gender gap when it comes to selfreporting sensitive skin, especially younger adults. This suggests potential changes in population skin characteristics and attitudes to self-reported skin sensitivity. These changes can be attributed to a higher consumption of cosmetic products that can be symptom triggers. Furthermore, the increased marketing of cosmetic products suitable for sensitive skin, particularly for men, may have increased consumer awareness of sensitive skin. ${ }^{19}$ The age-related reduction in self-reported skin sensitivity has similarly been observed in studies conducted in China and Japan.,20 This particular trend may be attributed to changes in skin properties, including reduction in tactile sensitivity and reduced irritability responses in older age. Such changes have been observed in skin irritation studies. ${ }^{21,22}$

Ethnicity was not fully analysed in this study due to the small numbers that were recruited. It is important to research different ethnic populations in the future, which could provide important clues into further understanding this complex condition. This is highlighted by the current research that observes an increased reporting of sensitive skin in Fitzpatrick phototype I to III and prior research that suggests sun sensitivity in the aetiology of sensitive skin. ${ }^{23,24}$ A further potential limitation is that data regarding cosmetic procedures, such as laser treatment, fillers, and botulinum toxin injections were not collected as part of the study. The interaction between these factors and sensitive skin is therefore unknown.

Differences in skin sensitivity between skin types suggest that barrier impairment may play a role in sensitive skin aetiology. Barrier function defects can lead to the activation of keratinocytes and Langerhans cells, inflammatory mediator release and the exposure of sensitive nerves, which can trigger vascular hyper-reactivity and sensory perceptions such as burning, stinging similar to that experienced in sensitive skin. ${ }^{25}$ Participants reporting oily, or combination skin types, more frequently identified as having sensitive skin, in contrast to the dry/normal skin types that more frequently reported non-sensitive skin. Further investigation to establish the difference between dry skin type and normal skin type and to establish an association between barrier function and skin sensitivity is required.

Current smokers also reported a higher frequency of sensitive skin; however, non-smokers, who had a previous history of smoking, reported similar rates of skin 
sensitivity as participants who had never smoked. This is suggestive of a reversible mechanism. Interestingly, (although not statistically significant), those with higher pack-years and longer periods of smoking reported a lower frequency of skin sensitivity, which suggests that initial exposure to smoking may trigger skin sensitivity with a gradual development of tolerance. Smokers have a thicker stratum corneum and decreased skin permeability, ${ }^{26}$ which may take years of exposure to develop, and this could explain the development of tolerance.

People within the self-reported sensitive skin group were more likely to report the presence of a concomitant dermatological condition, the most commonly reported skin condition being dry skin. This, again, hints at an aetiology focused around altered barrier function or heightened vascular reactivity. Increased baseline TEWL has been observed in atopic dermatitis and associated with an increased susceptibility to irritants. Furthermore, positive results from stinging tests have been more frequently observed in subjects with the condition. ${ }^{27} \mathrm{~A}$ personal or family history of eczema, hay fever or asthma reported sensitive skin more frequently, similar to the results reported by Willis, suggesting an immune-mediated component to this condition. Although all of the symptoms and signs linked with sensitive skin were reported significantly more when compared with those without sensitive skin, symptomology analysis did not present a clear pattern of disease characteristics. This suggests that patients may react differently depending on any given number of triggering factors, thus reinforcing the complexity of this condition. However, given the strong differentiation from the non-sensitive group, the results of this research are intended to be used as the foundations for the development of a clinically meaningful screening tool. This tool can aid patients, dermatologists, and those in the cosmetic industry to identify skin sensitivity in consumers and patients more consistently.

\section{Conclusion}

Evaluation between a self-reported sensitive skin cohort against a non-sensitive skin cohort has allowed for common inherent and external triggers to be distinguished between the groups in a large study, and to the authors' knowledge the first of its' kind using a general UK population. The key symptoms and signs of this syndrome identified in the literature were confirmed to be reported significantly more when compared with those without sensitive skin. However, no correlation or pattern of symptomology could be identified, which reinforcing the complexity of this condition. Given the strong differentiation from the non-sensitive group, the results of this research could be utilised for the development of a clinically meaningful screening tool for use in cosmetics trials.

\section{Funding}

This research was funded by Reckitt Benckiser Health Care Ltd. ("RB").

\section{Disclosure}

NF, VL, NS, ET and ABS are employees of Reckitt Benckiser Health Care Ltd. ("RB"). AN was an employee of RB at the time the study was conducted. RB manufactures dermatological products. The authors report no other conflicts of interest in this work.

\section{References}

1. Misery L, Loser K, Ständer S. Sensitive skin. 30th ed. J Eur Acad Dermatol Venereol. 2016;30(2):2-8. doi:10.1111/jdv.13532

2. Xu F, Yan S, Wu M, et al. Self-declared sensitive skin in China: a community-based study in three top metropolises. $J$ Eur Acad Dermatol Venereol. 2021;3(27):370-375.

3. Kim YR, Cheon HI, Misery L, et al. Sensitive skin in Korean population: an epidemiological approach. Skin Res Technol. 2018;24 (2):229-234

4. Farage MA. How do perceptions of sensitive skin differ at different anatomical sites? An epidemiological study. Clin Exp Dermatol. 2009;34(8):e521-e530.

5. Farage MA, Miller KW, Wippel AM, et al. Sensitive skin in the United States: survey of regional differences. Fam Med Medi Sci Res. 2013;02(112):2.

6. Misery L, Boussetta S, Nocera T, et al. Sensitive skin in Europe. J Eur Acad Dermatol Venereol. 2009;23(4):376-381.

7. Guinot C, Malvy D, Mauger E, et al. Self-reported skin sensitivity in a general adult population in France: data of the SU.VI.MAX cohort. J Eur Acad Dermatol Venereol. 2006;20(4):380-390.

8. Vanoosthuyze K, Zupkosky PJ, Buckley K. Survey of practicing dermatologists on the prevalence of sensitive skin in men. Int J Cosmet Sci. 2013;35(4):388-393.

9. Misery L, Ständer S, Szepietowski J, et al. Definition of sensitive skin: an expert position paper from the special interest group on sensitive skin of the international forum for the study of itch. Acta Dermato Venereologica. 2017;97(1):4-6.

10. Farage MA. Are we reaching the limits or our ability to detect skin effects with our current testing and measuring methods for consumer products? Contact Dermatitis. 2005;52(6):297-303. doi:10.1111/ j.0105-1873.2005.00614.x

11. Jourdain R, Ode L, Bastien P, et al. Ethnic variations in self perceived sensitive skin: epidemiological survey. Contact Dermatitis. 2002;46 (3):162-169

12. Farage MA. The prevalence of sensitive skin. Front Med. 2019;6:98.

13. Misery L, Jean-Decoster C, Mery S, Georgescu V, Sibaud V. A new ten-item questionnaire for assessing sensitive skin: the Sensitive Scale-10. Acta Derm Venereol. 2014;94(6):635-639. 
14. Moher D, Liberati A, Tetzlaff J, et al. Preferred reporting items for systematic reviews and meta-analyses: the PRISMA statement. BMJ. 2009;339(jul211):b2535.

15. UKRIO, U.R.I.O. Good Practice in Research: Internet-Mediated Research; 1996. Version1.0.

16. R: A language and environment for statistical computing. $R$ Core Team (2020). Vienna, Austria: $\mathrm{R}$ Foundation for Statistical Computing; 2020. Available from: https://www.R-project.org/. Accessed August 17, 2021.

17. Misery L, Jourdan E, Huet F, et al. Sensitive skin in France: a study on prevalence, relationship with age and skin type and impact on quality of life. J Eur Acad Dermatol Venereol. 2018;32(5):791-795.

18. Maibach HI, Lev-Tov H. The sensitive skin syndrome. Indian J Dermatol. 2012;57(6):419-423.

19. Farage MA, Maibach HI. Sensitive skin: closing in on a physiological cause. Contact Dermatitis. 2010;62(3):137-149.

20. Kamide R, Misery L, Perez-Curell N, et al. Sensitive skin evaluation in the Japanese population. J Dermatol. 2013;40(3):177-181.

21. Farage MA. Does sensitive skin differ between men and women? Cutan Ocul Toxicol. 2010;29(3):153-163.
22. Robinson MK. Population differences in acute skin irritation responses. Contact Dermatitis. 2002;46(2):86-93.

23. Misery L, Sibaud V, Merial-Kieny C, et al. Sensitive skin in the American population: prevalence, clinical data, and role of the dermatologist. Int J Dermatol. 2011;50(8):961-967.

24. Jourdain R, Ode L, Bastien P, Maibach HI. Ethnic variations in self-perceived sensitive skin: epidemiological survey. Contact Dermatitis. 2002;46(3):162-169.

25. Farage MA, Katsarou A, Maibach HI. Sensory, clinical and physiological factors in sensitive skin: a review. Contact Dermatitis. 2006;55:1-14.

26. Sandby-Møller J, Poulsen T, Wulf HC. Epidermal thickness at different body sites: relationship to age, gender, pigmentation, blood content, skin type and smoking habits. Acta Derm Venereol. 2003;83 (6):410-413.27.

27. Xu F, Yan S, Wu M, et al. Self-declared sensitive skin in China: a community-based study in three top metropolises. J Eur Acad Dermatol Venereol. 2013;27(3):370-375.

\section{Publish your work in this journal}

Clinical, Cosmetic and Investigational Dermatology is an international, peer-reviewed, open access, online journal that focuses on the latest clinical and experimental research in all aspects of skin disease and cosmetic interventions. This journal is indexed on CAS.
The manuscript management system is completely online and includes a very quick and fair peer-review system, which is all easy to use. Visit http://www.dovepress.com/testimonials.php to read real quotes from published authors. 